\title{
Identification of teleost Thy-1 and association with the microdomain/ lipid raft reggie proteins in regenerating CNS axons
}

\author{
Soeren-Oliver Deininger, ${ }^{\mathrm{a}, 1}$ Lawrence Rajendran, ${ }^{\mathrm{b}}$ Friedrich Lottspeich, ${ }^{\mathrm{c}}$ Michael Przybylski, ${ }^{\mathrm{a}}$ \\ Harald Illges, ${ }^{\mathrm{b}, \mathrm{d}}$ Claudia A.O. Stuermer, ${ }^{\mathrm{e}, *}$ and Alexander Reuter ${ }^{\mathrm{e}}$ \\ a Department of Chemistry, University of Konstanz, 78457 Konstanz, Germany \\ ${ }^{\mathrm{b}}$ Department of Biology, Immunology, University of Konstanz, 78457 Konstanz, Germany \\ c Max Planck Institute for Biochemistry, 82152 Martinsried, Germany \\ ${ }^{\mathrm{d}}$ Biotechnology Institute Thurgau, 8274 Tägerwilen, Switzerland \\ e Department of Biology, Neurobiology, University of Konstanz, 78457 Konstanz, Germany
}

\begin{abstract}
During regeneration, retinal ganglion cell axons in fish upregulate a cell surface protein that is recognized by the monoclonal antibody (mAB) M802. M802 antigen appeared to be linked to the intracellular, membrane-associated lipid raft/microdomain proteins reggie-1 and reggie-2 that were previously shown to be reexpressed in axon-regenerating neurons [Development 124 (1997), 577]. Here, we report the isolation of the M802 antigen and its identification as the teleost homolog of mammalian Thy-1. Fish Thy-1 is detected in the same detergent-insoluble lipid raft fractions from a fibroblast cell line and from axon regenerating retinae as reggie- 1 and 2 . Importantly, mAB M802 coimmunoprecipitates reggie- 1 and 2 from this lipid raft fraction, implying that fish Thy- 1 and reggies interact. This correlates with their colocalization in growing cell processes after M802 antigen/Thy-1 activation with mAB M802. These findings suggest a role of clustered M802 antigen/Thy-1 in reggie raft microdomains for cell growth and axon regeneration.
\end{abstract}

\section{Introduction}

Neurons that successfully regenerate axons after a lesion must be able to coordinate expression and assembly of growth-related proteins for growth cone formation, elongation, and navigation to distant targets. It appears that the response of neurons in the mammalian CNS to such a lesion is incomplete (Fawcett, 2001), whereas fish neurons readily reactivate this entire cellular program for repair and recovery of function (Benowitz et al., 2001). For instance, when the optic nerve is damaged, retinal ganglion cells (RGCs) in fish upregulate a range of growth-associated molecules (Benowitz and Lewis, 1983; LaBate and Skene, 1989; Stuermer and Leppert, 2001; Petrausch et al., 2000) including cell

\footnotetext{
* Corresponding author. Fax: +49-7531-88-3894.

E-mail address Claudia.Stuermer@uni-konstanz.de (C.A.O. Stuermer).

${ }^{1}$ Present address: Bruker Daltonik GmbH, 04318 Leipzig, Germany.
}

surface and intracellular proteins, whereas just a fraction of RGCs reexpresses a subset of those proteins in mammals (Jung et al., 1997; Benowitz et al., 2001; Petrausch et al., 2000). Although many of the lesion-induced proteins have been identified at the molecular level, the dynamics of their interaction that ultimately effect axon regeneration remains elusive.

Among the molecular components reexpressed by lesioned RGCs is a protein termed M802 antigen, whose regulated expressional pattern is being revealed by the monoclonal antibody (mAB) M802 (Stuermer et al., 1992; Schulte et al., 1997). M802 antigen appears associated with two intracellular proteins - which we named reggie-1 and reggie-2-insofar as mAB M802 led to the immunoisolation of reggies from fish larvae (Schulte et al., 1997). The same proteins were published independently as flotillin-2 and 1, respectively (Bickel et al., 1997). Reggie-1 and reggie-2 are colocalized and distributed in a punctate pattern along the cytoplasmic face of the plasma membrane where 
they define noncaveolar microdomains/lipid rafts (Lang et al., 1998; Stuermer et al., 2001a). Reggies/flotillins are evolutionarily highly conserved (Galbiati et al., 1998; Malaga-Trillo et al., 2002) and occur in a wide range of cells, including neurons and nonneuronal cells.

Lipid rafts/microdomains are conceived to act as platforms for the dynamic assembly of interacting proteins, especially of GPI-anchored cell surface and intracellular signal transduction proteins, such as nonreceptor tyrosine kinases of the src family to, thus, increase signal transduction efficacy (Simons and Toomre, 2000). Thus, lipid rafts promote signaling through the focal assembly of GPI-anchored proteins (Bruckner et al., 1999), which on their own have no direct access to intracellular signal transducing proteins (Simons and Ikonen, 1997; Friedrichson and Kurzchalia, 1998). Our own work in mammalian neurons, i.e., dorsal root ganglia and PC12 cells as well as in the lymphocytic Jurkat cells has shown the emergence of coclusters of the activated GPI-anchored immunoglobulin superfamily proteins F3 and Thy-1 in microdomains defined by reggie-1 and reggie-2 and has demonstrated the interaction of the GPI-anchored proteins with the reggies and fyn in coimmunoprecipitation assays (Stuermer et al., 2001a). These findings, together with the sensitivity of mAB M802 staining on goldfish RGC axons and CAR cells to PI-PLC (phosphatidylinositol specific phospholipase C, which cleaves GPI-anchors) suggested that M802 antigen is a GPI-anchored protein that interacts with reggie raft/microdomains.

The most widely used approach to determine raft association of a given protein of interest takes advantage of the fact that proteins residing in rafts are insoluble in cold non-ionic detergents and emerge in the detergent-insoluble glycolipid-rich membrane fractions (DIGs) after sucrose density centrifugation (Simons and Toomre, 2000). Appearance in DIGs identifies proteins from a mixture of cells or tissues as raft constituents yet does not necessarily imply an interaction of the proteins of this fraction, particularly as distinctly different types of raft/microdomains exist in different cells and even side by side in the same cell (Schnitzer et al., 1995; Kasahara and Sanai, 2000; Sowa et al., 2001; Stuermer et al., 2001a; Salzer et al., 2002). To determine the association of a given GPI-anchored protein with specific $\mathrm{raft} /$ microdomains requires colocalization of the proteins of interest at the microscopic level and, if possible, coimmunoprecipitation to show they interact (Simons and Toomre, 2000; Lang et al., 1998; Stuermer et al., 2001a).

That reggie-1 and 2 are noncaveolar raft constituents (Bickel et al., 1997; Solomon et al., 2002), are localized to growing axons from the mammalian and fish nervous system (Lang et al., 1998), are reexpressed in goldfish RGCs after nerve lesion (Schulte et al., 1997), and are linked to the M802 antigen points to a role of both reggie raft/microdomains and M802 antigen in axon growth and regeneration. mAB M802, made against proteins from regenerating goldfish optic nerves, stains all regenerating RGC axons throughout their path to the tectum as well as developing axons from newborn RGCs in unlesioned normal fish (Schulte et al., 1997), thus showing a pattern known from other growth-related proteins (Benowitz and Lewis, 1983; Vielmetter et al., 1991; Stuermer and Leppert, 2001), yet, it was difficult to determine its identity.

Here, we report the identification of the M802 antigen as fish Thy-1 and its close association with reggie in lipid rafts. We present evidence that both M802 antigen/Thy-1 and reggies are in DIGs/lipid raft fractions obtained from axonregenerating retinae and goldfish fibroblasts, that Thy- 1 is coimmunoprecipitated with reggie-1 and reggie- 2 from DIGs, and that Thy-1 is colocalized with reggie-2 in fibroblasts and RGC axons and growth cones. This may imply that reggie raft/microdomains and M802 antigen/Thy-1 play a role during RGC axon growth and regeneration.

\section{Results}

\section{mAB M802 staining on regenerating RGC axons}

mAB M802 produced a staining pattern reminiscent of many other growth-associated proteins in the fish visual pathway (Benowitz and Lewis, 1983; Vielmetter et al., 1991; Schulte et al., 1997; reviewed in Stuermer and Leppert, 2001, and in Benowitz et al., 2001). In isolated retinae, mAB M802 immunostained all RGC axons by 5 days post lesion on the lesioned side and just a few on the unlesioned side (Fig. 1A and B), which represent the growing axons from new RGCs, which are continuously added at the margin of the goldfish retina (Vielmetter et al., 1991). Moreover, regenerating RGC axons from retinal explants in tissue culture are M802 positive and staining encompasses the growth cones and filopodia. When mAB M802 is applied to living axons, it produces a punctate surface labeling as result of $\mathrm{mAB}$-induced crosslinking of the antigen (Schulte et al., 1997), which is typical of GPI-anchored proteins (Friedrichson and Kurzchalia, 1998).

To confirm GPI-anchorage of the M802 antigen, live retina explants were exposed to PI-PLC to cleave GPIanchors. This treatment abolished mAB M802 staining whereas labeling with an antibody against the integral membrane protein L1/E587 antigen (Vielmetter et al., 1991) persisted (Fig. 1C-F).

\section{Immunoaffinity purification of the M802 antigen and its identification as Thy-1}

Western blots with mAB M802 and proteins of goldfish brain extracts yielded-under nonreducing conditions-a prominent band at about $50 \mathrm{kDa}$ and a weaker one at 30 $\mathrm{kDa}$. The $30 \mathrm{kDa}$ increased in intensity in membrane preparations from retinae, in particular after nerve transection (Fig. 2A), which correlates with the upregulation of $\mathrm{mAB}$ M802 immunolabeling on all regenerating RGC axons. The 

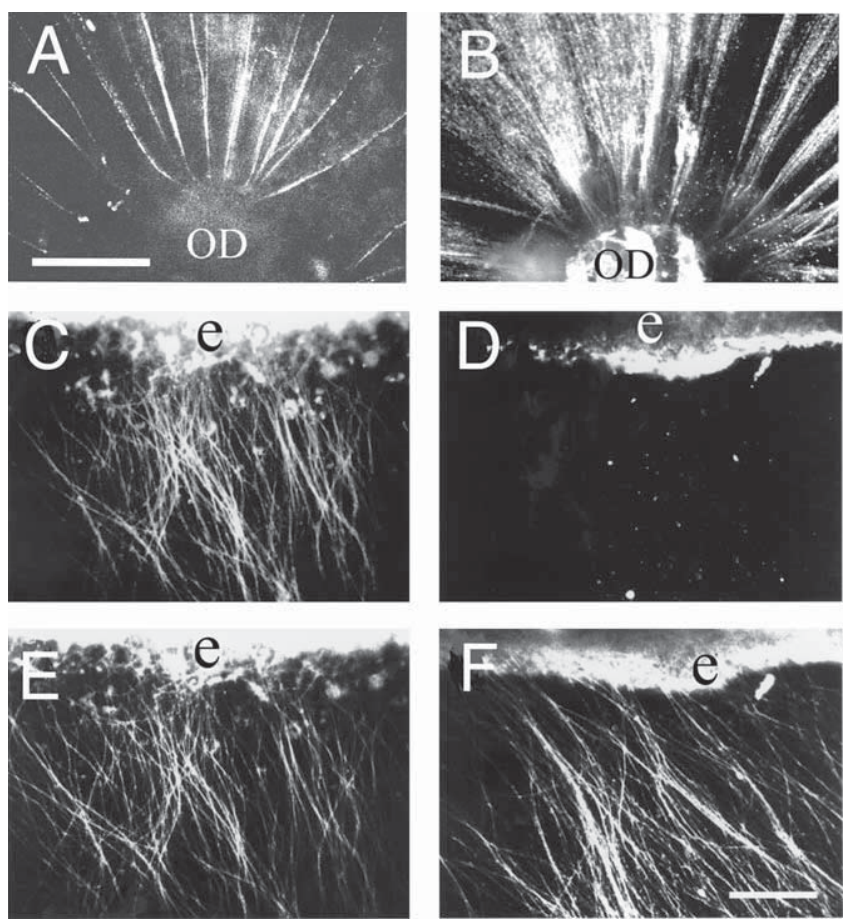

Fig. 1. Monoclonal antibody (mAB) M802 immunostaining on growing retinal ganglion cell (RGC) axons. Unfixed retinal wholemounts (A and B) and retinal explants (C-F) labeled by mAB M802 (A-D) and L1 (E and F). Retinal explants were untreated (C and $\mathrm{E}$ ) or treated with phosphatidylinositol specific phospholipase C (Pl-PLC) to cleave GPl-anchors (D and F). In the normal retina, mAB M802 binds selectively to the young RGC axons (A), whereas after optic nerve section all axons are stained (B). (C) RGC axons extending from retinal explants (e) in vitro are labeled by the $\mathrm{mAB}$ M802. (D) mAB M802 staining is no longer detectable when axons were treated with PI-PLC. (E) mAB M802 stained axons (from C) were double labeled with a polyclonal antibody against the E587/L1-like integral membrane protein. (F) The PI-PLC treated and M802-negative axons (from D) were labeled by the polyclonal antibody against E587 antigen/L1. OD, optic disc. Scale bars: $200 \mu \mathrm{m}$.

same protein fractions contained the 48-kDa reggie- 1 and reggie-2 proteins detected by their respective antibodies (Fig. 2A) but without showing this increase in intensity in the lesioned versus nonlesioned retina, most likely because of the expression of reggies in all retinal cells (and not just RGCs).

As it was not feasible to isolate the M802 antigen from retinae or brain, the goldfish fibroblast CAR cell line was used instead, which expressed this $30-\mathrm{kDa}$ protein at significantly higher concentrations. With approximately $1 \mathrm{mg}$ of antibody coupled to NHS-activated Sepharose, 20 pmol of M802 antigen was immunoaffinity-purified, which appeared as a broad 30-kDa band in Western blots (Fig. 2 B and $\mathrm{C}$ ), suggesting a substantial degree of protein glycosylation.

To remove N-linked glycan chains, PNGase F was added to the protein solution, resulting in additional bands between 17 and $30 \mathrm{kDa}$ in Western blots (data not shown), but the $30-\mathrm{kDa}$ protein persisted as the major form. We then applied an in-gel-deglycosylation procedure (compare Ex- perimental methods), and only then did the 17-kDa band emerge as the predominant form. The three fainter bands above the $17-\mathrm{kDa}$ core protein can be attributed to partially glycosylated M802 antigen (Fig. 2B), and suggest that the M802 antigen carries at least three N-linked glycan chains.

When the immunoaffinity-purified M802 antigen was exposed to PI-PLC and run in parallel with untreated protein, subsequent Western blot analysis revealed its presence in the untreated, but not in the PI-PLC-treated fraction (Fig. 2C). This confirms that the M802 antigen must be a GPIlinked protein.

To obtain partial amino acid sequences of the M802 antigen, our first approach consisted in subjecting the intact immunoaffinity-purified protein to in-gel digestion, but it was essentially resistant to proteolysis. We then subjected the purified in-gel-deglycosylated antigen to tryptic in-gel digestion and subsequent Edman sequencing and identified five peptide sequences (Fig. 3B).

Database searches (PBLAST) revealed a high homology of those five peptide sequences to several zebrafish expressed sequence tags (ESTs) with homologies to mammalian Thy-1. This information allowed us to assemble the complete cDNA sequence from several partial EST se-
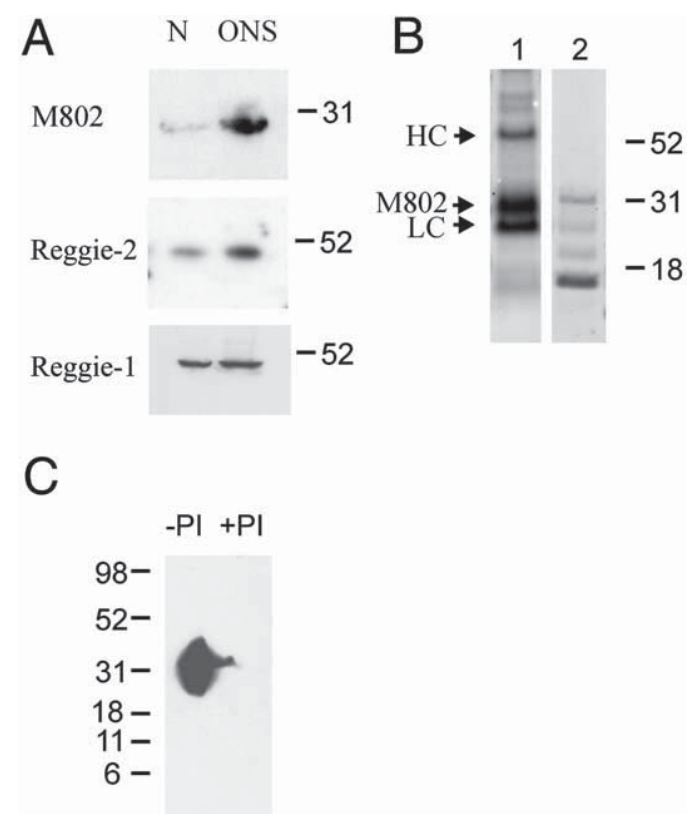

Fig. 2. Western blots of M802 antigen/Thy-1 and reggie. (A) Western blot analyses with mAB M802 and proteins from membranes of normal retinae (N) and retinae 4 weeks after optic nerve section (ONS) show an increase in M802/Thy-1 immunoreactivity after lesion. Both reggie-1 and reggie-2 show only a slight elevation compared to control (N). (B) Lane 1: Detection of the approximately 30-kDa immunoaffinity-purified M802 antigen/ Thy-1. HC, heavy chain, LC, light chain. Lane 2: M802 antigen/Thy-1 after in-gel-deglycosylation. The emergence of altogether 4 bands (between 17 and $31 \mathrm{kDa}$ ) indicates that the M802 antigen is heavily glycosylated and that the unglycosylated backbone is about $17 \mathrm{kDa}$ in size.(C) Phosphatidylinositol specific phospholipase C (PI-PLC) treatment of M802 antigen/ Thy-1. Western blot of untreated M802 antigen/Thy-1 (lane 1) and after PI-PLC treatment (lane 2). -PI, no PI-PLC treatment, +PI, PI-PLC treatment. 
A

1 ATGTTGTGCTACACCGCCTTTGCCACTCTCTTTTTACTTGGTGTTGTTAC 51 TGCCCAGACATCCCTTAGGATTACATCCTGCTTGACTAAAGACCAAAACC 101 TTCAAATGTCCTGCACCTTCACTCCAGCTCCAGACACCAAGCTCTCAAAA 151 ACATGCTACTACATGACTGACAATAAATTGATTGGCAGTACCAACAGCAG 201 CAGCACACCAGACAGCACGTTCAGGAACCGTGCCAATGTCACCATTACTG 251 ATAACAAGTGTGACCTGTATTTGAAGGGTTTACCAGATAGCAAACCTGCA 301 AACTACACTTGCTTTATCAGACAGACTGCCGCACCAGTGTCAATCATTCA 351 AACTGTGGACAAGAGTAAGCTTCAGACCTGTTCAGCGTGGAGTGTTTTGC 401 AACACAGTGGAGTAGCGTTCCTGTTGGGCTTCCTCACTTTCCCACTGTTG 451 TCAGAGCTTCTGTGA 465

50

100

150

200

250

300

350

400

450

\section{B}

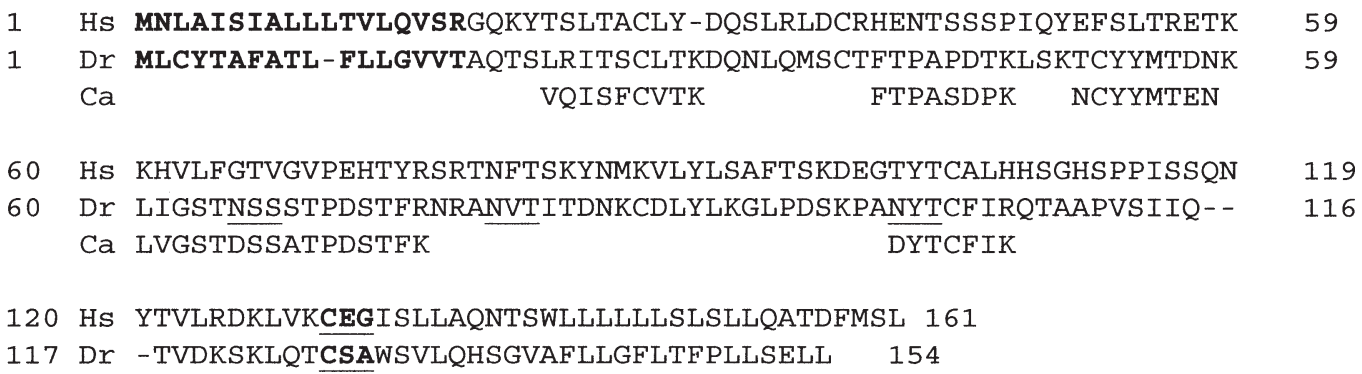

Fig. 3. The zebrafish Thy-1 cDNA and amino acid sequence and comparison to the Homo sapiens Thy-1. (A) The Danio rerio Thy-1 cDNA sequence was obtained by aligning the ESTs (expressed sequence tags) a1882694, be557611, be557676, bi476874, bi707266, bi843571, bi979664, bi979899, bm080899, and bm082075. The coding sequence of 465 base pairs was amplified by polymerase chain reaction from Danio rerio brain cDNA and sequenced. (B) Alignment of the Thy-1 precursor protein sequences from human (Hs) and zebrafish (Dr) and the peptides obtained from the goldfish (Ca) M802 antigen/Thy-1. Boldface, signal peptide; underscored, putative N-linked glycosylation sites; boldface and underscored, GP1 modification site. Hs, Homo sapiens; Dr, Danio rerio; Ca, Carassius auratus.

quences and to clone it by reverse transcription-polymerase chain reaction (Fig. 3A). This resulted in the identification of the zebrafish Thy-1 precursor protein (accession no. AY 157574). The zebrafish Thy-1 gene (Fig. 3B) is predicted to encode a glycoprotein of 154 amino acids, somewhat shorter than its human homolog of 161 amino acids. Similar to its human homolog, zebrafish Thy-1 is predicted to be synthesized as a precursor protein with an N-terminal signal sequence (17 amino acids compared to 18 in human [Seki et al., 1985]) and has a predicted GPI-anchor modification site at position 127 (position 130 in human). In addition, three putative N-linked glycosylation sites are contained in zebrafish Thy-1, and two of them are most likely also present in the amino acid sequence obtained from goldfish. Here, the asparagine residues $(\mathrm{N})$ are replaced by aspartic acid (D) as a result of the PNGase F treatment, which can lead to such changes (Fig. 2B).

The goldfish and zebrafish peptide sequences show 70\% identity at the protein level. The zebrafish Thy-1 precursor sequence shows $26 \%$ identity and $41 \%$ similarity to the human (Fig. 3B) and 30\% identity and 44\% similarity to the chick Thy-1 precursor molecule on the amino acid level.

mAB M802 detects goldfish Thy-1, but, unfortunately, fails to recognize the zebrafish Thy-1 homolog. To decide whether the identified zebrafish gene is indeed transcribed in axon-regenerating RGCs, wholemount in situ hybridization experiments were performed with retinae of unilaterally nerve transected zebrafish. In correspondence with the increase of mAB M802 staining in the goldfish retina, Thy-1 mRNA was upregulated in axon-regenerating zebrafish RGCs 2 weeks after nerve transection (Fig. 4B) and was not detected in corresponding regions of the normal control retina (Fig. 4C).

Association of goldfish Thy-1 with lipid raft/microdomains defined by reggie-1 and reggie- 2

To determine if M802 antigen/Thy-1 is associated with plasma membrane microdomains/rafts and, in particular, with reggie raft/microdomains, we prepared DIG-fractions 
A

B
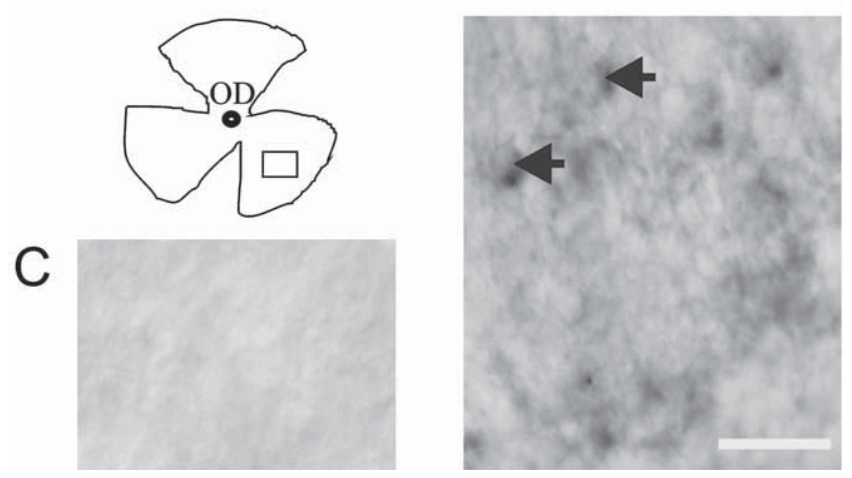

Fig. 4. In situ hybridization with a Thy-1 cRNA probe on zebrafish retina. (A) Schematic overview of a flattened retina wholemount. OD, optic disc; the rectangle indicates the selected area for B and C. (B) The in situ hybridization signal appears in many retinal ganglion cells in the (left) retina from a zebrafish 2 weeks after unilateral nerve transection (arrows), whereas no such signal can be detected in the corresponding regions of the normal (right) retina (C) from the same fish. Scale bar: $20 \mu \mathrm{m}$.

from CAR cells and goldfish retinae. Reggie- 1 and 2 were almost exclusively confined to the DIG/raft fractions 2-4 from CAR cells (Fig. 5A) and fractions 2-6 from retinae (Fig. 5B). M802 antigen/Thy-1 was found in the DIG/raft fractions $1-5$ of CAR cells and was, in addition, also present in the soluble fractions. This is consistent with the existence of mammalian Thy-1 in both raft and nonraft fractions (Sheets et al., 1997). The mAB M802-positive 30-kDa band also showed up in the retina raft fractions (3-4) but was less intense than in CAR cells, and only apparent in DIGs of the nerve transected retinae (Fig. 5B), which is attributed to the low M802 protein concentration. GAP43, which is known to be upregulated during RGC axon regeneration (Benowitz and Lewis, 1983; LaBate and Skene, 1989) and which associates with lipid rafts in hippocampal neurons (Ledesma et al., 1998), was also detected in DIGs/rafts from the axon-regenerating goldfish retina (Fig. 5B).

To determine whether M802 antigen/Thy-1 interacts with reggie, we performed coimmunoprecipitation experiments in DIG/raft fractions prepared from CAR cells, from which a sufficiently high protein concentration can be obtained. mAB M802 was used as the precipitating antibody and mouse IgG as control. Western blot analysis of immunoprecipitated proteins with mAB M802 and antibodies specific for reggie-1 and reggie-2, respectively, revealed the presence of M802 antigen/Thy-1 and of reggie-1 and reggie-2 in the precipitate (Fig. 5C). Such association did not appear in the nonraft soluble fractions, nor did the proteins appear in the mouse IgG control. These data demonstrate that an interaction between M802 antigen/Thy-1 with reggie-1 and 2 in CAR cell rafts indeed occurs.

To clarify if this association of M802 antigen/Thy-1 with reggie raft/microdomains is reflected by colocalization at
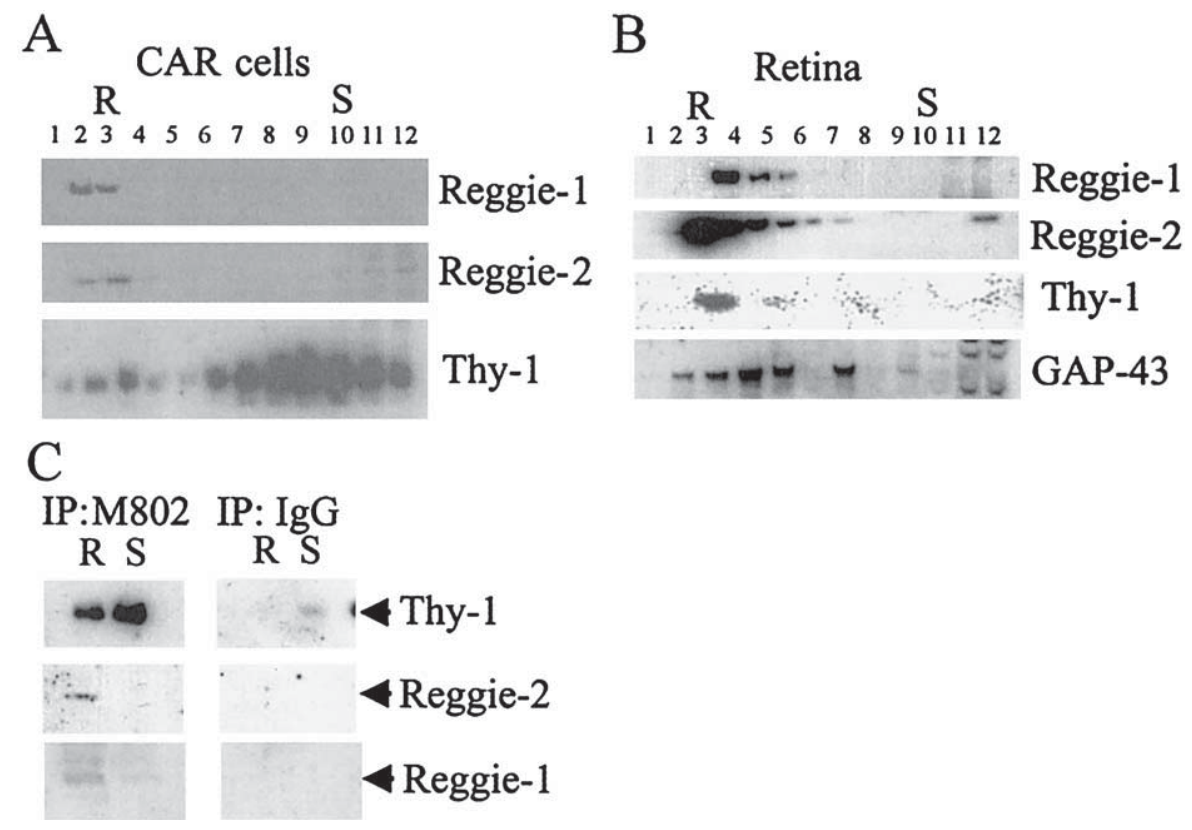

Fig. 5. Fish Thy-1 is associated with reggie lipid rafts. (A) In lipid raft (DIG) preparations from CAR cells, M802 antigen/Thy-1 is found in the raft fractions $1-6$ as well as in the nonraft fractions 7-12. Reggie-1 and reggie-2 are almost exclusively confined to the raft fractions (2-4). DIG, detergent-insoluble glycolipid-rich membrane fractions. (B) In retinae of nerve-transected goldfish, M802 antigen/Thy-1 is detectable in the lipid raft (DIG) fractions. Reggie-1, reggie-2, and GAP43 are localized predominantly in the raft fractions with some being present in the soluble fractions. (C) Coimmunoprecipitation of Thy-1, reggie-1, and reggie-2 by mAB M802 from lipid rafts. mAB M802 was used as the precipitating antibody. First panel: Western blot analysis of the M802 precipitate shows the presence of M802 antigen/Thy-1, reggie-1, and reggie-2 in lipid rafts, but not in the soluble fractions. Second panel: Unspecific IgG precipitation served as control (second panel). R, lipid raft; S, soluble fractions; Ip, immunoprecipitation. 
the microscopic level, we performed double immunostainings with mAB M802 in live CAR cells and RGC axons and with polyclonal reggie-2 antibody (after fixation) (Fig. 6). Anti-reggie-2 (Fig. 6, N) gave the typical punctate staining pattern known from our earlier studies, which have also shown that reggie-1 and reggie-2 are colocalized (Lang et al., 1998; Stuermer et al., 2001a). mAB M802, applied to living cells and axons, induced the formation of "clusters" of M802 antigen/Thy-1 and such "clustered rafts" are typical of GPI-anchored proteins activated by antibodies including Thy-1 (Friedrichson and Kurzchalia, 1998). Through activation by the mAB, M802 antigen/Thy-1 became preferentially accumulated at the motile rims and edges and filopodia-like protrusions of CAR cells (as has been observed in rat fibroblasts; Aarts et al., 1999), and the overlay of the images reveals a significant degree of colocalization of M802 antigen/Thy-1 and reggie-2 along the cellular periphery and its protrusions (Fig. 6A, B, and D-I). Colocalization of M802 antigen/Thy-1 and reggie-2 was not apparent in the remaining CAR cell plasma membrane since Thy-1 was shifted to the cell periphery, and was also less striking without mAB M802-induced clustering of M802 antigen/Thy-1 (Fig. 6M, N, and O). This does not exclude that an association and interaction of M802 antigen/Thy-1 and reggie exist in unstimulated cells; on the contrary, this interaction does occur as is evidenced by the outcome of the coimmunoprecipitation assays (Fig. 5). Yet, without crosslinking, GPI-anchored protein rafts are too small to be visualized microscopically in cultured cells (Varma and Mayor, 1998). Colocalization of activated M802 antigen/ Thy-1 and reggie-2 was also detectable along the regenerating RGC axons (Fig. 6C), and in particular, in RGC growth cones (Fig. $6 \mathrm{~J}-\mathrm{L}$ ) where the punctate distribution of the two proteins is more readily recognized. Therefore, goldfish M802 antigen/Thy-1 clusters in noncaveolar lipid rafts and colocalizes with reggie-2 preferentially after M802 antigen/Thy-1 activation, in regenerating RGC growth cones, CAR cell motile edges, and filopodia-like protrusions.

\section{Discussion}

During regeneration, RGC axons reexpress on their surface the M802 antigen/Thy-1 in association with the lipid $\mathrm{raft} /$ microdomain proteins reggie- 1 and reggie- 2 . As this suggested an interaction of these proteins, we have isolated the M802 antigen and identified it here as the teleost Thy-1 homolog. Our results not only demonstrate that M802 antigen/Thy- 1 is detected in the same lipid rafts/DIG-fractions that contain reggie- 1 and reggie-2, but show in addition that M802 antigen/Thy-1 is coimmunoprecipitated with reggie-1 and reggie-2 from this DIG-fraction. This implies that a functional interaction occurs between raft-associated M802 antigen/Thy-1 and reggie proteins. This is consistent with the concept on the role of rafts, which serve as platforms for the dynamic assembly of GPI-anchored proteins including Thy-1 to enhance signal transduction (Simons and Toomre, 2000), and specifically with the more recent insights into the role of reggies, which demarcate a specific type of raft/ microdomains distinct of caveolae (Lang et al., 1998; Stuermer et al., 2001a). The association of Thy-1 with reggie rafts is corroborated by the present microscopic evidence showing that activated M802 antigen/Thy-1 colocalizes with reggie- 2 in RGC axons and growth cones and in the edges of CAR cells. This indicates that interactions of Thy-1 with reggie occur in motile cellular processes and may contribute to aspects of growth. Taken together, these findings imply that M802 antigen/Thy-1 in association with reggies might play a role during axon regeneration in fish (Schulte et al., 1997; Lang et al., 1998) and suggest that the ability of neurons to restore plasma membrane microdomains as centers for the assembly of GPI-anchored proteins including Thy-1 is important for axon regrowth.

\section{Identification of teleost Thy-1}

Our earlier attempts to identify the M802 antigen/Thy-1 from the fish CNS have proven difficult mainly because the amount of protein recovered from immunoisolation attempts was always too low for sequencing and because it emerged as a weaker $30 \mathrm{kDa}$ and a stronger $50-\mathrm{kDa}$ protein band in Western blots with brain-derived proteins. These difficulties were overcome by using the CAR cells, which express M802 antigen/Thy-1 at a much higher level than brain tissue and by employing improved biochemical procedures. In fact, the purified M802 antigen/Thy-1 resisted proteolytic digestion due to its high amount of glycosylation, a feature known from mammalian Thy-1 (Williams and Gagnon, 1982). Prerequisite for partial peptide sequencing was, therefore, another methodical step involving ingel-deglycosylation of the recovered M802 antigen/Thy-1. The peptides isolated from goldfish and the predicted sequence from cDNA cloning of zebrafish Thy-1 exhibit 26\% homology to higher vertebrate Thy-1.

Despite its low sequence similarity, the presence of Thy-1 in fish and the preservation of structural features (e.g., signal peptide, immunoglobulin domain, GPI-anchor, and glycosylation) suggests a possibly conserved role across all vertebrate lineages. That Thy- 1 is associated with reggies, which are evolutionarily highly conserved in mammalian and teleost cellular systems (Malaga-Trillo et al., 2002), is in line with the hypothesis that the function of Thy-1 might depend - at least in part-on its interaction with reggie raft/microdomains (Simons and Toomre, 2000; Stuermer et al., 2001a).

\section{Interaction of Thy-1 with reggie-1 and reggie-2}

That fish Thy-1 was successfully coimmunoprecipitated with reggie- 1 and reggie- 2 by mAB M802 from CAR-cell 

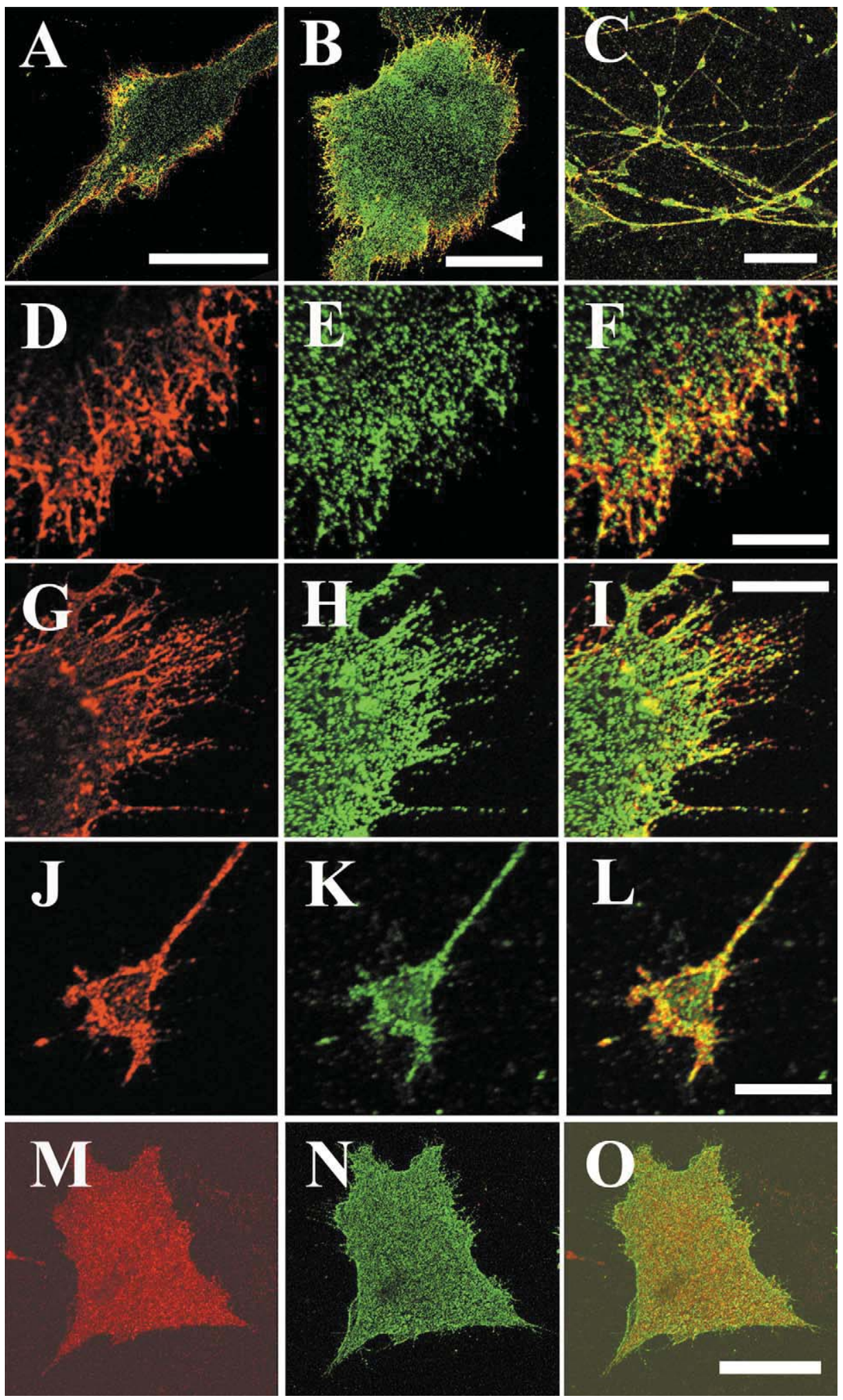

Fig. 6. Double immunostaining with mAB M802 and polyclonal anti-reggie-2. (A, B, D-I, and M-O) CAR cells. (C) RGC axons in culture. (J-L) RGC growth cone; mAB M802 staining appears in red, anti-reggie-2 in green. (A-C and F, I, L, and O) Overlay of red/green (yellow). RGC, retinal ganglion cell. When live CAR cells (A, B, and D-I) are exposed to mAB M802, a punctate pattern emerges that accumulates in the edges of the cell and in the filopodia-like protrusions (A, B, D, and G). Double labeling with an anti-reggie-2 shows a typical punctate pattern across the entire cell, including its edges and filopodia (A, B, E, H, and N). The overlay (A, B, F, and I) reveals colocalization (yellow) in the membraneous edges and in the filopodia-like protrusions. The arrow in B denotes the region shown in higher magnification in D-F. In RGC axons (C) and growth cones (J-L), live exposure to mAB M802 (J) and subsequent reggie-2 antibody staining (K) results in substantial colocalization of the two proteins (C and L). Without antibody-induced activation, the M802 antigen/Thy-1 is distributed over the entire cell (without condensation along the edges, $\mathrm{M}$ ). Reggie-2 is likewise found across the cell, but its distribution is punctate $(\mathrm{N})$ and colocalization is less extensive (O). Scale bars: $50 \mu \mathrm{m}(\mathrm{A}-\mathrm{C}$, and $\mathrm{O}$ ) and $10 \mu \mathrm{m}(\mathrm{F}, \mathrm{I}$, and $\mathrm{L})$. 
DIG-fractions shows that the GPI-anchored surface Thy-1 protein and reggies at the cytoplasmic face of plasma membrane functionally interact. Thy-1, which does not span the plasma membrane and has on its own no direct access to the intracellular signal transduction machinery, acquires thereby intracellular partners capable of recruiting, for instance, fyn (Stuermer et al., 2001a). In T-lymphocytic Jurkat cells, Thy-1 activation by antibody crosslinking induces the cell-specific reaction known as "capping," which leads to the accumulation of Thy-1, the T-cell receptor components, reggie-1 and 2, and signal transducing molecules to one aspect ot the cell (Janes et al., 1999; Stuermer et al., 2001a) and promotes their interaction. Thy-1 interaction with the reggies may also be relevant for CAR cells. The shift of Thy-1 to the cell periphery upon application of mAB M802 to the cells somehow resembles the capping dynamics of Thy- 1 in T cells, and may suggest that M802 antigen/ Thy-1 has a function in regulating cell motility. This may also apply to RGCs and their regenerating axons, since Thy- 1 is colocalized with reggie- 2 along RGC axons and in their growth cones (where reggie-2 is coclustered with reggie-1; Lang et al., 1998). Moreover, fish Thy-1 and the reggies occur in the same DIG-fraction obtained from axonregenerating retinae, which is no proof, but in light of their colocalization another strong indication for their interaction.

The protein complex consisting of M802 antigen/Thy-1, reggie-1, and reggie-2 was only found in the raft fraction and was not apparent in the soluble fraction. The distributional pattern of the reggies and M802 antigen/Thy-1 in DIGs of retinae shows that only a fraction of M802 antigen/ Thy- 1 is raft associated. This is in accordance with findings of Jacobson and co-workers who have shown that GPIassociated proteins as Thy- 1 associate with rafts only transiently (Sheets et al., 1997) and the raft residence time varies according to the cell types and cell activation state (Dietrich et al., 2001). This would be consistent with the view that M802 antigen/Thy-1 enters the reggie rafts upon activation and participates in lipid raft-based signal transduction thereby contributing to cell specific responses, i.e., growth and regeneration. The growth-associated protein GAP43, which is also localized in lipid rafts (Laux et al., 2000) and whose synthesis is also upregulated during regeneration (Benowitz and Lewis, 1983; LaBate and Skene, 1989), has been proposed to play a role in regulating actinbased structures and motility (Laux et al., 2000). Whether and exactly how M802 antigen/Thy-1 and its association with reggies regulates growth remains to be investigated, for instance, by morpholino knockdown strategies in zebrafish, which efficiently block protein translation (Corey and Adams, 2001), and by identification of the relevant signal transduction molecules.

The finding that mAB M802 detects an additional band of about $50 \mathrm{kDa}$ in Western blots with brain-derived proteins suggests that M802 antigen/Thy-1 occurs in this tissue in dimeric or multimeric forms, as has been reported for mammalian Thy-1 (Bonewald et al., 1984; Mahanthappa and Patterson, 1992). Or, M802 antigen/Thy-1 might be relatively stably associated with another protein of yet unknown identity. It is conceivable that this multimeric form reflects a functional state of Thy-1 typical for these tissues or their physiological state, which might correlate with the activated state of cells in vitro induced by the application of Thy-1 antibodies.

This sort of activation leading to "clustered rafts" (Simons and Toomre, 2000) is prerequisite to visualize the colocalization of Thy-1 with reggies at the light microscopical level (Stuermer et al., 2001a) and is thought to mimick ligand binding with the consequence that the recruitment of intracellular signal transducing proteins is enhanced (Simons and Ikonen, 1997; Stefanova et al., 1991). Interactions of Thy- 1 and reggie appear to occur without this massive stimulation and may have a modulatory effect.

In spite of decades of research, the function of Thy- 1 in the nervous system remains elusive (Morris, 1992). In higher vertebrates, Thy- 1 has been discussed as having both positive and negative effects on axon growth. Thy-1 somehow functions as a receptor for astrocyte-associated ligands and this interaction inhibits axon growth (Tiveron et al., 1992; Morris, 1992; Lipton et al., 1992). Other studies suggest that antibody-induced crosslinking of Thy-1 causes an increase in $\mathrm{Ca}^{2+}$ influx through specific $\mathrm{Ca}^{2+}$ channels, which enhances neurite outgrowth (Doherty et al., 1993), possibly involving a modulation of GAP43 (Aarts et al., 1999). Thy-1 knockout mice have no apparent phenotype (Morris, 1992), and no increase in axon regeneration or plasticity was observed in the absence of Thy- 1 in a more recent study (Barlow et al., 2002). Reports testing its role during regeneration and repair show that Thy- 1 is downregulated after lesion. This was observed after retinal insults like ischemia or NMDA injection (Nash and Osborne, 1999) and after optic nerve crush (Perry et al., 1984; Schlamp et al., 2001). In fact, Thy-1 mRNA levels decreased rapidly after nerve crush in mice and declined to nearly undetectable levels. This is in marked contrast to the situation in fish, where, according to our earlier and present results, M802 antigen/Thy-1 is upregulated after optic nerve lesion on both mRNA (zebrafish) and protein level (goldfish), leading to the speculation that Thy-1 reexpression upon lesion may contribute to the remarkable regeneration capabilities of RGC axons in fish.

Thus, the upregulation of M802 antigen/Thy-1 and reggies in axon-regenerating RGCs, their association in lipid rafts, and cocluster formation in regenerating growth cones predict a raft microdomain-based function in axon regrowth. Altogether, this supports the view that microdomain-associated multimolecular assemblies form along the plasma membrane during axon regeneration and are most likely functionally involved in recovery, growth, or regeneration. 


\section{Experimental methods}

\section{Preparation of goldfish vagal lobe and retina membranes}

Regions rich in M802 antigen/Thy-1 are the vagal lobes and the retina after nerve transection. Freshly excised goldfish vagal lobes or retinae were frozen in liquid nitrogen and stored at $-80^{\circ} \mathrm{C}$ prior to preparation. Aliquots of vagal lobes or retinae were ground up in liquid nitrogen by using a mortar and pestle. The proteins were then treated with homogenization buffer (10 mM Tris, $1 \mathrm{mM}$ spermidine, 2 $\mathrm{mM} \mathrm{CaCl}_{2}$, Complete Inhibitor [Roche], $\mathrm{pH}$ 7.4) and loaded on a sucrose step gradient (50-20\% sucrose in phosphatebuffered saline [PBS]). After centrifugation at $10,000 \mathrm{~g}$ the membrane fractions were recovered from the interface between the $20 \%$ and 50\% sucrose layers, rinced in PBS, and extracted in lysis buffer (20 mM Tris, $150 \mathrm{mM} \mathrm{NaCl}, 1 \%$ Triton X-100, $0.5 \%$ deoxycholate, $0.1 \%$ sodium dodecyl sulfate [SDS], $1 \mathrm{mg} / \mathrm{ml}$ bovine serum albumin [BSA], $\mathrm{pH}$ 7.6) in the presence of Complete Inhibitor by sonication on ice for $60 \mathrm{~min}$. The solution was centrifuged at 100,000 and the clear membrane extract was collected.

\section{Preparation of CAR cell lysate}

CAR cells (a goldfish fibroblast cell line; American Type Culture Collection, Manassas, VA, USA) were maintained in F12 medium with $10 \%$ fetal calf serum at $27^{\circ} \mathrm{C}$, removed from the culture flask with a rubber policeman, centrifuged at $400 \mathrm{~g}$, and immersed in lysis buffer (30 min on ice) in an ultrasound bath. Solid particles were removed by centifugation at maximal speed in an Eppendorf centrifuge and the supernatant was collected.

\section{Immunoaffinity purification of M802 antigen}

mAB M802 was coupled to NHS-activated Sepharose 4B Phast Flow Beads (Amersham Pharmacia Biotec, Freiburg, Germany) at a concentration of $10 \mu \mathrm{g}$ of antibody/ $\mu$ l of beads. Vagal lobe and retina membrane extract or CAR cell lysate was incubated with mouse IgG Beads (Sigma; $10 \mu \mathrm{l}$ beads/200 $\mu$ l lysis buffer) for preclearing and incubated at $4^{\circ} \mathrm{C}$ for $4 \mathrm{~h}$ by rolling over top. Beads were removed by centrifugation and discarded. mAB M802 beads were added ( $20 \mu \mathrm{l}$ beads per culture flask CAR cells and $20 \mu \mathrm{l}$ of beads per 100 vagal lobes or 8 retinae, respectively) and incubated overnight at $4^{\circ} \mathrm{C}$ by rolling over top.

The supernatant was removed and beads were subsequently washed with lysis buffer (without BSA), Tris-buffered saline (TBS) buffer $+1 \%$ TritonX-100, TBS buffer with $900 \mathrm{mM}$ $\mathrm{NaCl}+1 \%$ TritonX-100, TBS buffer $+1 \%$ TritonX-100, and preelution buffer (TBS $+0.2 \%$ dodecylmaltoside). Beads were transferred to a column and eluted dropwise with elution buffer $(0.2 \%$ dodecylmaltoside in $0.1 \%$ Trifluoroactic acid (TFA). Antigen containing fractions were identified by dotblotting and probing with mAB M802.

\section{PI-PLC treatment of the M802 antigen}

Aliquots of the M802 antigen from vagal lobes (with $0.16 \%$ Nonidet P-40 [NP-40]) were dialyzed against $50 \mathrm{mM}$ triethanolamine, $10 \mathrm{mM}$ EDTA, $\mathrm{pH} 7.5$, and exposed to 0.5 U PI-PLC (Sigma) or to buffer alone. Both aliquots were incubated at $37^{\circ} \mathrm{C}$ overnight, lyophilized, and probed by Western blotting.

\section{Deglycosylation of M802 antigen}

\section{In solution}

M802 antigen was dialyzed against PBS; 20 units of PNGase F (Roche), dialyzed against PBS with 1\% n-octylglycoside, was added to the M802 antigen solution, which was also dialyzed against PBS. After overnight incubation at $37^{\circ} \mathrm{C}$, the antigen was detected by Western blotting.

\section{In gel deglycosylation}

M802 antigen was lyophilized and redissolved in SDS sample buffer for alkylation (0.4 M Tris, 1\% SDS, $10 \mathrm{mM}$ EDTA, Orange G, 26 mM DTT; Westermeier, 1990) and incubated at $96^{\circ} \mathrm{C}$ for $5 \mathrm{~min}$. After cooling down, $1 / 10$ vol of a $20 \%(\mathrm{wt} / \mathrm{vol})$-iodoacetamine solution was added and incubated at room temperature (RT) for $30 \mathrm{~min}$. The sample was run on a Novex $4-12 \%$ gradient gel according to the manufacturer's instructions (Novex) and stained with colloidal Coomassie Blue. The M802-positive band was excised, destained with $10 \%$ acetic acid, 30\% ethanol, washed with water for $10 \mathrm{~min}$, and shrunk by addition of $70 \%$ acetonitrile. The excess solution was removed and the gel pieces dried in a Speedvac; $50 \mu$ l of PNGase F solution containing 2.5 units of the enzyme was prepared in PBS with $20 \mathrm{mM}$ EDTA and 1\% n-octylglycoside. The dried gel pieces were slowly rehydrated with the PNGase F solution and incubated overnight at $37^{\circ} \mathrm{C}$. After washing with water, the slices were dehydrated by addition of acetonitrile, dried in the Speedvac, rehydrated with Novex NuPAGE Sample Buffer (Invitrogen), and homogenized in an Eppendorf tube. The slurry was transferred to a NuPAGE $4-12 \%$ gel and reelectrophoresed.

\section{Edman sequencing of M802 antigen partial peptides}

M802 antigen, prepared as described above, was subjected to gel electrophoresis. The M802 positive band was excised and subjected to in-gel-digestion with endoproteinase LysC and endoproteinase AspN. Deglycosylated M802 antigen/Thy-1 was in-gel-digested with trypsin. Partial peptides were separated by high performance liquid chromatography and subjected to Edman sequencing reactions.

\section{Immunolabeling}

For double immunolabeling, CAR cells were grown on fibronectin-coated coverslips and retinal explants on poly- 
lysine/laminin-coated coverslips. Both were exposed to mAB M802 (1:100) for $30 \mathrm{~min}$ at RT. The secondary antibody (Cy3-coupled polyclonal goat anti-mouse; 1:1000) was used for crosslinking (10 $\mathrm{min}$ at RT). The cells were fixed for 5 min in methanol $\left(-20^{\circ} \mathrm{C}\right)$, rinsed, and blocked with $1 \%$ BSA and $10 \%$ donkey serum in PBS-T. The cells were incubated with the second primary polyclonal antibody against reggie-2 (R659, 1:100; Stuermer et al., 2001a) overnight, washed, and incubated with the secondary antibody (Alexa green coupled polyclonal donkey anti-rabbit; $1: 1000$ ) for $1 \mathrm{~h}$ at RT. After several rinses with PBS, the cells were embedded in a mixture of $5 \mathrm{~g}$ of Mowiol 4-88 (Hoechst), $20 \mathrm{ml}$ of PBS, and $10 \mathrm{ml}$ of glycerol, and analyzed by confocal laser scanning microscopy (Zeiss). For PI-PLC treatment, retinae were isolated, attached to nylon membranes (Vielmetter et al., 1991), and treated with $1 \mathrm{U}$ PI-PLC per milliliter of F12 medium for $2 \mathrm{~h}$ at $27^{\circ} \mathrm{C}$. After washing with PBS, the retinae were stained essentially as CAR cells (for M802 staining) and as described before (for E587 staining; Vielmetter et al., 1991).

\section{Preparation of detergent-insoluble fractions}

DIGs were prepared essentially as described before (Solomon et al., 2002). In brief, the CAR cells or goldfish retinae were homogenized in $10 \mathrm{mM}$ Tris buffer ( $\mathrm{pH}$ 7.2) containing $1 \%$ Triton X-100 and treated with a Wheaton douncer. The homogenate was layered on a 5-40\% discontinuous sucrose gradient and centrifuged at 200,000 $\mathrm{g}$ in a SV50.1 rotor for $16 \mathrm{~h}$ at $4^{\circ} \mathrm{C}$ in a Beckman ultracentrifuge. Fractions of $400 \mu \mathrm{l}$ were collected from the top of the gradient and used for subsequent analysis.

\section{Immunoprecipitation}

The DIG fractions were precleared with protein G-coupled magnetic beads (Dynal) for $2 \mathrm{~h}$ at $4^{\circ} \mathrm{C}$ and subjected to magnetic separation. Protein G-coupled magnetic beads were incubated with mAB M802 or with mouse IgG antibodies as control for $50 \mathrm{~min}$ at RT. The beads were washed twice with $0.1 \%$ NP-40 lysis buffer, added to the precleared fractions, and incubated overnight at $4^{\circ} \mathrm{C}$. The antibodybound beads were magnetically separated and washed $1 \times$ with $1 \%$ NP-40 lysis buffer and $3 \times$ with $0.1 \%$ NP-40 lysis buffer. The immunoprecipitated proteins were analyzed by Western blotting.

\section{Western blotting}

Immunoprecipitated proteins were separated under reducing or nonreducing (for M802 staining) conditions in SDS-polyacrylamide gel electrophoresis. The separated proteins were blotted onto nitrocellulose membranes according to standard procedures and blocked twice with 1.5\% BSA in TBST (10 mM Tris, $150 \mathrm{mM} \mathrm{NaCl,} \mathrm{0.02 \%} \mathrm{Tween)}$ at RT. The blots were then incubated with the primary antibodies for either $1 \mathrm{~h}$ at RT or overnight at $4^{\circ} \mathrm{C}$, washed (6×, $10 \mathrm{~min})$, and then incubated with the respective secondary antibodies conjugated with peroxidase for $45 \mathrm{~min}$ at RT. After an additional three washes, detection was performed with the Supersignal chemiluminescence kit (Pierce).

\section{Optic nerve transection and retina preparation}

Goldfish and zebrafish were maintained in the central animal facilities (TFA, University of Konstanz). The optic nerves of adult goldfish (5-8 cm long) and zebrafish (3 months old) were cut under MS 222 (Sigma) anesthesia in compliance with animal welfare legislation. Retinae were isolated and attached to nylon membranes (Vielmetter et al., 1991), placed on polylysine/laminin-coated coverslips, and incubated in F12 medium (with $12 \%$ fetal calf serum) in humidified chambers at $28^{\circ} \mathrm{C}$.

\section{In situ hybridization}

Isolated retinae of adult zebrafish were mounted on Hybond $\mathrm{N}^{+}$membranes (Amersham) and subjected to a wholemount in situ hybridization procedure based on the Boehringer DIG system essentially as described before (Lang et al., 1998). The probes were full-length sense and antisense cRNAs of zebrafish Thy-1. All in situ hybridizations were run as doublets with antisense cRNA and with sense cRNA as control. Only antisense reactions resulted in staining; the sense controls were void.

\section{Acknowledgments}

This study was supported by grants of the DFG (Deutsche Forschungsgemeinschaft), the BMBF (Bundesministerium für Bildung, Forschung und Wissenschaft), and the FCI (Fonds der Chemischen Industrie) to C.A.O.S. and by a grant from the Ministry of Science and Culture of the State of Baden-Württemberg to H.I. We thank U. Binkle and M. Wiechers for excellent technical assistance.

\section{References}

Aarts, L.H.J., Verkade, P., van Dalen, J.J.W., van Rosen, A.J., Gispen, W.H., Schrama, L.H., Schotman, P., 1999. B-50/GAP-43 potentiates cytoskeletal reorganization in raft domains. Mol. Cell. Neurosci. 14, 85-97.

Barlow, J.Z., Kelley, K.A., Bozdagi, O., Huntley, G.W., 2002. Testing the role of the cell-surface molecule Thy-1 in regeneration and plasticity of connectivity in the CNS. Neuroscience 111, 837-852.

Benowitz, L.I., Leon, S., Tabibiazar, R., Jing, J., Irwin, N., 2001. Axonal regeneration in the primary visual pathway of goldfish and rats, in: Ingoglia, N.A., Murray, M. (Eds.), Axonal Regeneration in the Central Nervous System, Marcel Dekker Inc., New York, NY, pp. 45-66. 
Benowitz, L.I., Lewis, E.R., 1983. Increased transport of 44000- to 49000dalton acidic proteins during regeneration of the goldfish optic nerve: a two-dimensional gel analysis. J. Neurosci. 3, 2153-2163.

Bickel, P.E., Scherer, P.E., Schnitzer, J.E., Oh, P.M., Lisanti, P., Lodish, H.F., 1997. Flotillin and epidermal surface antigen define a new family of caveolae-associated integral membrane proteins. J. Biol. Chem. 272, 19793-19802.

Bonewald, L., Ades, E.W., Tung, E., Marchalonis, J.J., Wang, A.C , 1984. Biochemical characterization of human Thy-1. J. Immunogenet. 11, 487-497.

Bruckner, K., Pablo Labrador, J., Scheiffele, P., Herb, A., Seeburg, P.H., Klein, R., 1999. EphrinB ligands recruit GRIP family PDZ adaptor proteins into raft membrane microdomains. Neuron 22, 511-524.

Corey, D.R., Adams, J.M., 2001 Morpholino antisense oligonucleotides: tools for investigating vertebrate development. Genome Biol. 2, Reviews 2015.

Dietrich, C., Volovyk, Z.N., Levi, M., Thompson, N.L., Jacobson, K., 2001. Partitioning of Thy-1, GM1, and cross-linked phospholipid analogs into lipid rafts reconstituted in supported model membrane monolayers. Proc. Natl. Acad. Sci. USA 98, 10642-10647.

Doherty, P., Singh, A., Rimon, G., Bolsover, S.R., Walsh, F.S., 1993. Thy-1 antibody-triggered neurite outgrowth requires an influx of calcium into neurons via N- and L-type calcium channels. J. Cell Biol. 122, 181-189.

Fawcett, J.W., 2001. Intrinsic control of regeneration and the loss of regenerative ability in development. in: Ingoglia, N.A., Murray, M. (Eds.), Axonal Regeneration in the Central Nervous System. Marcel Dekker Inc., New York, NY, pp. 161-184.

Friedrichson, T., Kurzchalia, V., 1998. Microdomains of GPI-anchored proteins in living cells revealed by crosslinking. Nature 394, 802-805.

Galbiati, F., Volonte, D., Goltz, J.S., Steele, Z., Sen, J., Jurcsak, J., Stein, D., Stevens, L., Lisanti, M.P., 1998. Identification, sequence and developmental expression of invertebrate flotillins from Drosophila melanogaster. Gene 210, 229-237.

Janes, P.W., Ley, S.C., Magee, A.I., 1999. Aggregation of lipid rafts accompanies signaling via the T cell antigen receptor. J. Cell Biol. 147, 447-461.

Jung, M., Petrausch, B., Stuermer, C.A., 1997. Axon-regenerating retinal ganglion cells in adult rats synthesize the cell adhesion molecule L1 but not TAG-1 or SC-1. Mol. Cell. Neurosci. 9, 116-131.

Kasahara, K., Sanai, Y., 2000. Functional roles of glycosphingolipids in signal transduction via lipid rafts. Glycoconj. J. 17, 153-162.

LaBate, M., Skene, J.H.P., 1989. Selective conservation of Gap-43 structure in vertebrate evolution. Neuron 3, 299-310.

Lang, D.M., Lommel, S., Jung, M., Ankerholt, R., Petrausch, B., Laessing, U., Wiechers, M.F., Plattner, H., Stuermer, C.A.O., 1998. Identification of reggie-1 and reggie-2 as plasma membrane-associated proteins which cocluster with activated GPI-anchored cell adhesion molecules in non-caveolar micropatches in neurons. J. Neurobiol. 37, 502-523.

Laux, T., Fukami, K., Thelen, M., Golub, T., Frey, D., Caroni, P., 2000. GAP-43, MARCKS, and CAP23 modulate PI(4,5)P2 at plasmalemmal rafts, and regulate cell cortex actin dynamics through a common mechanism. J. Cell Biol. 149, 1455-1471.

Ledesma, M.D., Simons, K., Dotti, C.G., 1998. Neuronal polarity: essential role of protein-lipid complexes in axonal sorting. Proc. Natl. Acad. Sci. USA 95, 3966-3971.

Lipton, S.A, Leifer, D , Barnstable, C J., 1992. Selectivity of Thy-1 monoclonal antibodies in enhancing neurite outgrowth. Neurosci. Lett. 137, 75-77.

Mahanthappa, N.K., Patterson, P.H., 1992. Thy-1 multimerization is correlated with neurite outgrowth. Dev. Biol. 150, 60-71.

Malaga-Trillo, E., Laessing, U., Lang, D.M., Meyer, A., Stuermer, C.A., 2002. Evolution of duplicated reggie genes in zebrafish and goldfish. J. Mol. Evol. 54, 235-245.

Morris, R.J., 1992. Thy-1, the enigmatic extrovert on the neuronal surface. BioEssays 14, 715-722.

Nash, M.S., Osborne, N.N., 1999. Assessment of Thy-1 mRNA levels as an index of retinal ganglion cell damage. Invest. Ophthalmol. Vis. Sci. 40, 1293-1298.
Perry, V.H., Morris, R.J., Raisman, G., 1984. Is Thy-1 expressed only by ganglion cells and their axons in the retina and optic nerve? J. Neurocytol. 13, 809-824.

Petrausch, B., Jung, M., Leppert, C., Stuermer, C.A.O., 2000. Lesioninduced regulation of netrin receptors and modification of netrin-1 expression in the retina of fish and grafted rats. Mol. Cel. Neurosci. 16, 350-364.

Salzer, U., Hinterdorfer, P., Hunger, U., Borken, C., Prohaska, R., 2002. $\mathrm{Ca}(++)$ dependent vesicle release from erythrocytes involves stomatin-specific lipid rafts, synexin (annexin vii), and sorcin. Blood 99, 2569-2577.

Schlamp, C.L., Johnson, E.C., Li, Y., Morrison, J.C., Nickells, R.W., 2001. Changes in Thy-1 gene expression associated with damaged retinal ganglion cells. Mol. Vision 7, 192-201.

Schnitzer, J.E., McIntosh, D.P., Dvorak, A.M., Liu, J., Oh, P., 1995. Separation of caveolae from associated microdomains of GPI-anchored preins. Science 269, 1435-1439.

Schulte, T., Paschke, K.A., Laessing, U., Lottspeich, F., Stuermer, C.A.O., 1997. Reggie-1 and reggie-2, two cell surface proteins expressed by retinal ganglion cells during axon regeneration. Development 124, 577-587.

Seki, T., Spurr, N., Obata, F., Goyert, S., Goodfellow, P., Silver, J., 1985. The human Thy-1 gene: structure and chromosomal location. Proc. Natl. Acad. Sci. USA 82, 6657-6661.

Sheets, E.D., Lee, G.M., Simson, R., Jacobson, K., 1997. Transient confinement of a glycosylphosphatidylinositol-anchored protein in the plasma membrane. Biochemistry 36, 12449-12458.

Simons, K., Ikonen, E., 1997. Functional rafts in cell membranes. Nature 387, 569-572.

Simons, K., Toomre, D., 2000. Lipid rafts and signal transduction. Nat. Rev. Mol. Cell Biol. 1, 31-39.

Solomon, S., Masilamani, M., Rajendran, L., Bastmeyer, M., Stuermer, C.A.O., Illges, H., 2002. The lipid raft-microdomain-associated protein reggie-1/flotillin-2 is expressed in human $B$ cells and is localized at the plasma membrane and centrosome in PBMCs. Immunobiology 205, 108-119.

Sowa, G., Pypaert, M., Sessa, W.C., 2001. Distinction between signaling mechanisms in lipid rafts vs. caveolae. Proc. Natl. Acad. Sci. USA 98, 14072-14077.

Stefanova, I., Horejsi, V., Ansotegui, I.J., Knapp, W., Stockinger, H., 1991. GPI-anchored cell surface molecules complexed to protein tyrosine kinases. Science 254, 1016-1019.

Stuermer, C.A.O., Bastmeyer, M., Bähr, M., Strobel, G., Paschke, K., 1992. Trying to understand axonal regeneration in the CNS of fish. J. Neurobiol. 23, 537-559.

Stuermer, C.A.O., Lang, D.M., Kirsch, F., Wiechers, M., Deininger, S.-O., Plattner, H., 2001a. Glycosylphosphatidyl-inositol-anchored proteins and fyn kinase assemble in noncaveolar plasma membrane microdomains defined by reggie-1 and -2. Mol. Biol. Cell. 12, 3031-3045.

Stuermer, C.A.O., Leppert, C.A., 2001b. Molecular determinants of retinal axon pathfinding in fish. in: Ingoglia, N.A., Murray, M. (Eds.), Axonal Regeneration in the Central Nervous System. Marcel Dekker Inc., New York, NY, pp. 19-44.

Tiveron, M.C., Barboni, E., Pliego Rivero, F.B., Gormley, A.M., Seeley, P.J., Grosveld, F., Morris, R.J., 1992. Selective inhibition of neurite outgrowth on mature astrocytes by Thy-1 glycoprotein. Nature 355, 745-748.

Varma, R., Mayor, S., 1998. GPI-anchored proteins are organized in submicron domains at the cell surface. Nature 394, 798-801.

Vielmetter, J., Lottspeich, F., Stuermer, C.A.O., 1991. The monoclonal antibody E587 recognizes growing (new and regenerating) retinal axons in the goldfish retinotectal pathway. J. Neurosci. 11, 3581-3593.

Westermeier, R, 1990. Elektrophoresepraktikum. VCH Verlagsgesellschaft, Weinheim.

Williams, A.F., Gagnon, J., 1982. Neuronal cell Thy-1 glycoprotein: homology with immunoglobulin. Science 216, 696-703. 